\title{
Hepatic cystic echinococcosis due to Echinococcus granulosus, grossly observed by needle aspiration
}

\author{
Min Kyu Kang ${ }^{1}$, Kook Hyun $\mathrm{Kim}^{1}$, and Joon Hyuk Choi ${ }^{2}$
}

Departments of ${ }^{1}$ Internal Medicine and ${ }^{2}$ Pathology, Yeungnam University College of Medicine, Daegu, Korea
Received: April 25, 2018

Revised : May 3, 2018

Accepted: May 3, 2018

\section{Correspondence to}

Kook Hyun Kim, M.D.

Tel: +82-53-620-3576

Fax: $+82-53-654-8386$

E-mail: kimkh@yu.ac.kr
A 66-year-old man visited our hospital for the evaluation of bulging mucosa observed by endoscopy at a medical check-up. He had mild dyspepsia and abdominal discomfort, and a history of diabetes, hypertension, and coronary stent insertion due to previous myocardial infarction. He denied any history of travelling to the Middle East or contact with pets. Abdominal computed tomography and ultrasonography revealed an approximately $7 \mathrm{~cm}$ sized cystic lesion with wall enhancement (Fig. 1). Blood chemistry was as follows: white blood cells, 9,490/ $\mu \mathrm{L}$ (eosinophil, 16.1\%); hemoglobin, $12.9 \mathrm{~g} / \mathrm{dL}$; platelets, $284 \times 10^{3} / \mu \mathrm{L}$; serum albumin, 4.45 $\mathrm{g} / \mathrm{dL}$; serum bilirubin, $0.82 \mathrm{mg} / \mathrm{dL}$; serum aspartate aminotransferase, 26 IU/L; serum alanine aminotransferase, $53 \mathrm{IU} / \mathrm{L}$; and eosinophilic count, 1,510/

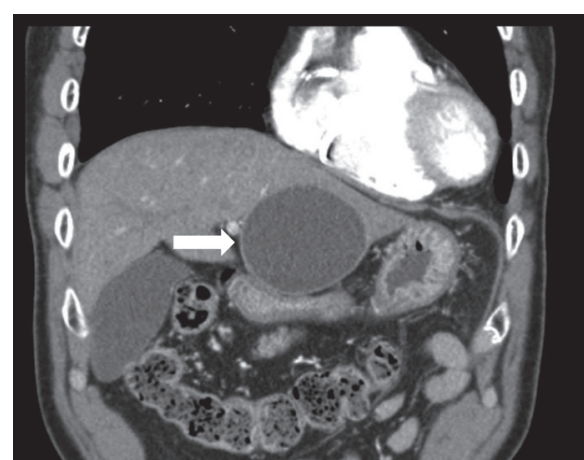

Figure 1. Abdominal computed tomography revealed about $7 \mathrm{~cm}$ sized, homogenous wall-enhanced, cystic lesion (white arrow) without inner significant solid component. $\mu \mathrm{L}$. Additional laboratory findings including stool protozoa, helminth ova, serum Paragonimus, and sparganum immunoglobulin $\mathrm{G}$ were all negative.

Cyst aspiration was planned for further analysis of the hepatic cystic lesion. Ultrasound guided percutaneous 18-gauge needle aspiration showed transparent cystic fluid with multiple white rice-granule sized pellets (Fig. 2A). Microscopic analysis confirmed those granules as cystic echinococcosis (CE) due to Echinococcus granulosus (Fig. $2 \mathrm{~B}$ and $2 \mathrm{C}$ ). We strongly recommended surgical resection of the cyst, but he refused and was subsequently lost to follow-up.

Human CE remains an endemic disease in developing countries, usually in regions of Central Asia, China, Russia, and the Middle East. Adult E. granulosus parasitizes the small intestine of dogs and eggs are excreted in feces. The ingested eggs hatch and penetrate the human small intestine and migrate to liver. However, the route of parasite infection was not clear in our case. Treatment options are surgery, chemotherapy with benzimidazole or percutaneous cyst aspiration.

The study was approved by the Institutional Review Board of Yeungnam University Medical Center (IRB No. YUMC 2018-12-034) and informed consent was waived by the IRB. 

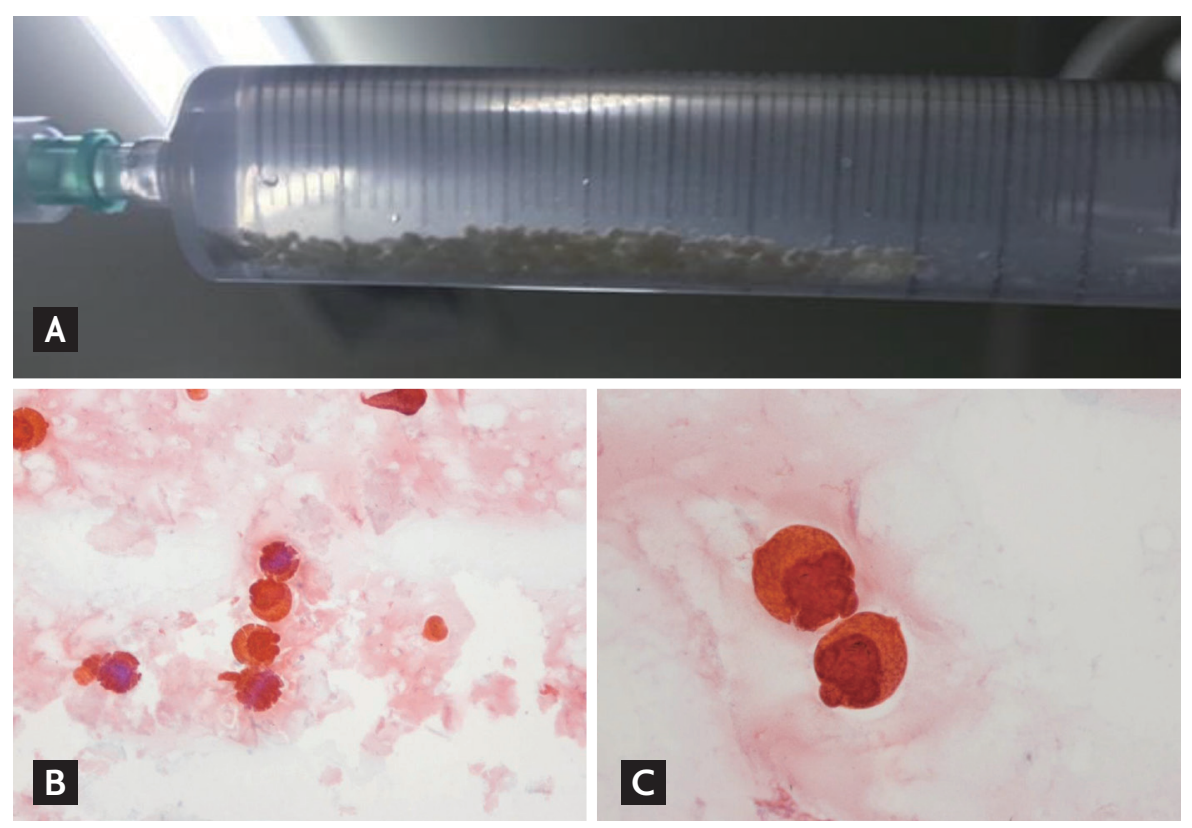

Figure 2. (A) Cystic fluid and materials by 18 -gauge needle aspiration, (B, C) various morphological features of Echinococcus granulosus (Papanicolaou stain, B: ×40; C: ×100, respectively).

\section{Conflict of interest}

No potential conflict of interest relevant to this article was reported.

\section{Acknowledgments}

This paper was supported by a 2016 research grant from Yeungnam University. 